\title{
An Observer Looks at Synchronization
}

\author{
Henk Nijmeijer and Iven M. Y. Mareels
}

\begin{abstract}
In the literature on dynamical systems analysis and the control of systems with complex behavior, the topic of synchronization of the response of systems has received considerable attention. This concept is revisited in the light of the classical notion of observers from (non)linear control theory.
\end{abstract}

Index Terms-Detectability, dynamical systems, observers, reduced order observers, synchronization.

\section{INTRODUCTION}

$\mathbf{I}$ $\mathrm{N}$ RECENT years there has been considerable interest in the dynamics and control of systems exhibiting complex behavior. The number of papers related to this subject seems to grow at an almost exponential rate [1]. For an admittedly already "dated" review of some of the prevailing research problems the reader may consult the seminal papers in [2].

The purpose of the present paper is to revisit the concept of synchronization from a mathematical control theoretic perspective. More specifically we want to explore how the observer notion from (non)linear control theory links in with synchronization. For an introduction to nonlinear control theory we refer to [4]-[6].

Synchronization, as introduced by Pecora and Carroll [7], [8] has been studied from various angles. Often a master-slave formalism is taken, e.g., [7]-[12]. Given a particular dynamical system, the master, together with an identical (sub)system, the aim is to synchronize to the master system the complete response of the slave system, by driving the latter with a (scalar) signal derived from the master system. In this context synchronization is often considered to be a remarkable property when the master dynamics are chaotic and thus sensitive to initial condition variations. A promising application in secure communication suggested in [9] and [40] uses such a chaotic master dynamics to mask a message and a synchronized slave system to recover the message.

The above master-slave viewpoint leaves some ambiguity as to what the actual slave system should be, given the master system. A naive, but often realistic approach, would be to consider the master dynamics (transmitter) as transmitting a signal to the slave dynamics (receiver) and the receiver is

Manuscript received January 15, 1997; revised June 7, 1997 and June 23, 1997. Most of this work was completed while H. Nijmeijer was visiting and I. M. Y. Mareels was a member of the Department of Engineering and the Cooperative Research Centre for Robust and Adaptive Systems, Australian National University, Canberra, Australia. This work was supported by the Cooperative Research Centre for Robust and Adaptive Systems by the Australian Government under the Cooperative Research Centres Program. This paper was recommended by Guest Editor M. P. Kennedy.

H. Nijmeijer is with the Department of Applied Mathematics, University of Twente, 7500AE Enschede, The Netherlands.

I. M. Y. Mareels is with the Department of Electrical and Electronic Engineering, The University of Melbourne, Parkville, Victoria 3052, Australia.

Publisher Item Identifier S 1057-7122(97)07615-0. requested to recover the full state trajectory of the transmitter. The problem is of course only interesting if the signal received is not equal to the full state. In this situation the receiver has in principle the freedom to build any dynamical system. The receiver system could be a copy of the master system, but it need not be. The real requirement is that given the received signal the receiver dynamics will synchronize to the transmitters' dynamics. In thus allowing the receiver the freedom of which dynamical system to implement, we enlarge the class of master-slave systems that allow synchronization. Note that at this point we do not consider the actual physical realization of the new receiver's dynamical system. In certain applications this may be crucial, but this aspect lies beyond the scope of the present paper.

The problem just described is closely related to the observer problem from control theory. For linear dynamical systems a complete solution to the problem is well known [13], [14]. For nonlinear systems a few partial results exist [15], [16], [26]-[28]. This observation is at the core of the paper. Another point that will transpire from the exposition is that the complexity of the dynamics involved is of little concern in our discussion of observer design or synchronization.

Besides the master-slave perspective on synchronization another viewpoint is expressed in [3]. There synchronization is seen as the design of a (feedback) mechanism for the receiver, using the transmitted signal, so as to ensure that the controlled receiver synchronizes with the transmitter. This approach to synchronization is in essence a control problem, which we do not discuss in this paper, but see [30].

A standard approach in solving the observer problem in control theory is to use as receiver a copy of the transmitter (of course with unknown initial state) modified with a term depending on the difference between the received signal and its prediction derived from the observer. The additional term aims at attenuating the difference between the state of the transmitter and the state of the observer system. This procedure may be shown to be successful in many instances, but certainly no global validity can be claimed. The synchronization problem requires one to establish global asymptotic stability for the zero solution of the error dynamics, the dynamics governing the difference between the transmitter state and observer state. Rigorous proofs often rely on Lyapunov arguments [22]-[24]. Most of the existing results concerning synchronization also rely on Lyapunov based arguments [9], [12], and [10].

That a solution to the above synchronization problem, or observer problem, may be feasible under certain conditions may be deduced from the Takens embedding theorem [17], which is closely related to the observability property for nonlinear dynamical systems [18], [19]. In essence the ob- 
servability property states that the history of the transmitted signal contains all the information required to reconstruct a state variable for the master dynamics. The observability property is a generic property of dynamical systems. However it falls short of implying the existence of an observer or receiver that synchronizes. In the case of linear systems the link between observability (or better detectability) and the existence of an observer can be made explicitly. However in the nonlinear context the situation is not that clear, and apart from some local results, cited before, few results are available.

Often, the observer complexity can be reduced by noting that the given transmitter signal already contains part of the state information. Hence, one only needs to reconstruct the complementary part of the transmitter state, that part which is not explicitly contained in the transmitted signal. This leads naturally to the concept of a reduced observer.

The presentation in the paper is kept simple. No attempt is made at stating the most general results available from (non)linear control theory. Appropriate references are provided. Also in order to avoid (sometimes nontrivial) complications, we assume throughout that all dynamical systems are defined on an open subset of an Euclidean space and have sufficient regularity such as to guarantee the existence of unique solutions. In this paper only dynamical systems described in continuous time are considered. A completely similar treatment for dynamical systems defined via maps is possible.

The observer perspective in approaching synchronization is to the best of our knowledge new.

The paper is organized as follows. In the next section we define the problem of synchronization in some detail and state clearly the standing assumptions. Sections II-V each revisit the observer problem for a specific subclass of systems. In Section VI we make some observations about the general problem.

\section{A. Preliminaries}

For more details we refer to [5].

$D h$ denotes the Jacobian of $h$, it is the matrix with entries $D h_{i j}$ being the partial derivative of the $i$ th component of $h$ with respect to the $j$ th argument of $h$.

By $D_{i} h$ we denote the partial derivative of $h$ with respect to its $i$ th argument.

Given a vector field $f$ and a scalar valued function $h$ define the (iterated) directional derivative of $h$ in the direction of $f$ as: $L_{f}^{k+1} h(x)=$ $L_{f}\left(L_{f}^{k} h(x)\right)$, with $L_{f}^{0} h(x)=h(x)$ and $L_{f} h(x)=$ $\Sigma_{i}\left(D_{i} h(x)\right) f_{i}(x)$.

$[X, Y]$ denotes the Lie bracket of two vector fields, it is defined as the vector field $[X, Y](x)=(D Y(x)) X(x)$ $(D X(x)) Y(x) \cdot a d_{X}^{k} Y$ is defined via $a d_{X}^{0} Y=Y, a d_{X} Y=$ $[X, Y]$ and $a d_{X}^{k+1} Y=\left[X, a d_{X}^{k} Y\right]$.

Given vector fields $X_{j}, j=1, \cdots$ we define as distribution the object $d=\operatorname{span}\left\{X_{j}, j=1, \cdots\right\}$. We call the distribution $d$ involutive if it is closed under the Lie bracket operation.

Vector fields live in the tangent space of the manifold under consideration. In this paper we consider dynamics over Euclidean space, hence the tangent space can equally be identified with Euclidean space. When discussing the dual space of the tangent space we refer to cotangent space, covector fields and codistribution.

In order to decide on the non existence of observers, the notions of observability and detectability are crucial. Consider the time invariant system

$$
\begin{array}{ll}
\dot{x}(t)=f(x(t)), & x(0)=x_{o} \in \mathbb{R}^{n}, \quad t \geq 0, \\
y(t)=h(x(t)), \quad y \in \mathbb{R}^{p} .
\end{array}
$$

Denote by $x\left(t, x_{o}\right)$ a solution to (1), i.e. $x\left(0, x_{o}\right)=x_{o}$ and $\dot{x}\left(t, x_{o}\right)=f\left(x\left(t, x_{o}\right)\right)$. Assume $f$ and $h$ to be smooth.

The system (1) is called locally observable at $x_{o}$ if for all initial conditions $x_{1}, x_{2}$ in some neighborhood $U$ of $x_{o}$ if $h\left(x\left(t, x_{1}\right)\right)=h\left(x\left(t, x_{2}\right)\right.$ for all $t$ such that $x\left(t, x_{1}\right), x\left(t, x_{2}\right) \in$ $U$ implies that $x_{1}=x_{2}$. The system (1) is called locally observable if it is locally observable at any $x_{o} \in \mathbb{R}^{n}$.

The observation space $O$ is the linear space over $\mathbb{R}$ of the vector fields $L_{f}^{k} h_{i}(x)$ for $i=1, \cdots, p$ and $k=0,1,2, \cdots$. A sufficient, and almost necessary, condition for local observability is that the codistribution $d O(x)=\operatorname{span}\left\{D L_{f}^{k} h_{i}(x)\right.$, $i=1, \cdots, p k=0,1, \cdots\}$ satisfies $\operatorname{dim} d O(x)=n, \forall x \in \mathbb{R}^{n}$.

In case $\operatorname{dim} d O(x)<n$, then the vector field leaves the kernel of the observability codistribution invariant (i.e., $[f, \operatorname{ker} d O] \subset \operatorname{ker} d O$ ). The $\operatorname{ker} d O$ is an involutive distribution. A necessary condition for the existence of a valid observer becomes that the dynamics of $f$ restricted to $\operatorname{ker}(d O)$ be asymptotically stable. In this case we say that the system is detectable.

Specializing to linear dynamics, $f(x)=A x$ and $h(x)=C x$ for appropriate matrices $A$ and $C$, detectability is expressed by:

$$
\text { if rank }\left(\begin{array}{c}
\lambda I-A \\
C
\end{array}\right)<n \text { then } \operatorname{Real}(\lambda)<0 \text {. }
$$

In the case that the matrix

$$
\left(\begin{array}{c}
\lambda I-A \\
C
\end{array}\right)
$$

has rank $n$ for all complex $\lambda$ we say that the system is observable. This condition for observability is equivalent to $\operatorname{dim} d O(x)=n, \forall x \in \mathbb{R}^{n}$, which in the linear case reduces to

$$
\operatorname{ker} O:=\operatorname{ker}\left(\begin{array}{c}
C \\
C A \\
\vdots \\
C A^{n-1}
\end{array}\right)=\{0\} \text {. }
$$

\section{Problem Statement}

We state two particular problems in the area of observer design, which are closely linked to synchronization. First we introduce the full observer, next the reduced observer problem.

Let us consider dynamics governed by:

$$
\dot{x}(t)=f(x(t), t), \quad x(0)=x_{0} \in \mathbb{R}^{n}, \quad t \geq 0 .
$$

We assume throughout that the vector field $f$ is smooth and that the system (5) has a unique solution $x\left(t, x_{0}\right)$ passing 
through the initial state $x\left(0, x_{0}\right)=x_{0}$ defined on an interval $\left(-T_{-},+\infty\right)$, with $T_{-}>0$.

The state $x\left(t, x_{0}\right)$ is not directly available, only an output is measured, say:

$$
y(t)=h(x(t), t) \in \mathbb{R}^{p}, p<n .
$$

We assume throughout that $h$ is smooth.

\section{A. Full Observer Problem}

Given the system description (5) with (6), a natural question is 'When is it possible to reconstruct the state $x$ from measurements of the output $y$ ?' This is the so called observability problem. Notice that this question is clearly aligned with the synchronization problem discussed in the introduction 'Given the signal $y(t)$ when can we synchronize the state of another dynamical system to $x(t)$, the state of (5)?'

A full observer for the system (5) with (6) is defined as

$$
\begin{aligned}
& \dot{\hat{x}}(t)=\hat{f}(\hat{x}(t), y(t), t), \quad \hat{x}(0)=\hat{x}_{0} \in \mathbb{R}^{n}, \quad t \geq 0 \\
& \hat{y}(t)=h(\hat{x}(t), t) \in \mathbb{R}^{p}
\end{aligned}
$$

where $\hat{x} \in \mathbb{R}^{n}$ and $\hat{f}$ is a smooth vector field, parameterized by $y$ and $t$, such that the error $e(t)=x(t)-\hat{x}(t)$ asymptotically converges to zero as $t \rightarrow \infty$ for all initial conditions $\hat{x}_{0}$ and $x_{0}$ and moreover if $e\left(t_{0}\right)=0$ then $e(t) \equiv 0$ for all $t \geq t_{0}$.

\section{B. Reduced Observer}

In a sense if we reconstruct the state via (7), we are reconstructing more information than necessary. Indeed the output $y$ already contains some information about the state which we need not reconstruct. To discuss this point further let us specialize to the case where the output equation does not depend explicitly on time, $y(t)=h(x(t))$. The time dependence in the output (6) leads to some complications we prefer to avoid.

More precisely let us assume that there exists a diffeomorphism $\phi: \mathbb{R}^{n} \rightarrow \mathbb{R}^{n}$ such that:

$$
\begin{aligned}
\phi(x) & =\left(\begin{array}{l}
h(x) \\
v(x)
\end{array}\right)=\left(\begin{array}{l}
y \\
z
\end{array}\right), \\
x & =\phi^{-1}(y, z) .
\end{aligned}
$$

Given the output $y$ it suffices to reconstruct $z$ in order to know $x$. Now $z$ is governed by the differential equation:

$$
\dot{z}(t)=f_{r}(z(t), y(t), t), \quad z(0)=v\left(x_{0}\right), \quad t \geq 0 .
$$

The vector field $f_{r}$ is defined as $f_{r}(z, y, t)=$ $D v\left(\phi^{-1}(y, z)\right) f\left(\phi^{-1}(y, z), t\right)$. This expression follows from (8) together with (5).

Let $\hat{z}$ be defined by:

$$
\dot{\hat{z}}(t)=f_{r}(\hat{z}(t), y(t), t), \quad \hat{z}(0)=\hat{z}_{0}, \quad t \geq 0 .
$$

If the diffeomorphism $\phi$ can be chosen such that the error $e_{r}(t)=z(t)-\hat{z}(t)$ converges to zero asymptotically as $t \rightarrow \infty$ and moreover if $e_{r}\left(t_{0}\right)=0$ implies that $e_{r}(t) \equiv 0$ for all $t \geq t_{0}$ then we call the system (10) a reduced observer for the nonlinear system (5).
Of course once a reduced observer is found the full state is asymptotically recovered via $x(t)=\phi^{-1}(y(t), \hat{z}(t))$.

Remark 1: In formulating the observer problem above, the links with synchronization are obvious. We emphasize the importance of the state space transformations allowed for in the reduced observer problem and the freedom to choose the vector field $\hat{f}$ in the full observer problem. This additional freedom, which appears to be largely lacking in the discussions on synchronization, enlarges considerably the class of systems for which the observer/synchronization problem can be addressed successfully.

\section{LINEAR SYSTEMS}

In the case of linear time invariant dynamics the problem of constructing a full or reduced observer is solved completely [13], [14].

The relevant (5) and (6) or (1) now simplify to

$$
\begin{array}{ll}
\dot{x}(t)=A x(t), & x(0)=x_{0}, \quad A \in \mathbb{R}^{n \times n} \\
y(t)=C x(t), & C \in \mathbb{R}^{p \times n} .
\end{array}
$$

For any matrix $A$ the solutions of (11) are defined on $(-\infty,+\infty)$, the observer problem is hence always well posed.

\section{A. Full Observer}

For the linear system (11) an observer system (7) takes on the form

$$
\begin{aligned}
& \dot{\hat{x}}(t)=A \hat{x}(t)+K(\hat{y}(t)-y(t)), \quad \hat{x}(0)=\hat{x}_{0} \\
& \hat{y}(t)=C \hat{x}(t) .
\end{aligned}
$$

Here $K \in \mathbb{R}^{n \times p}$ is known as an output injection matrix. The error $e(t)=x(t)-\hat{x}(t)$ is governed by

$$
\dot{e}(t)=(A+K C) e(t) .
$$

These dynamics represent a valid observer if a gain matrix $K$ can be found such that the matrix $A+K C$ has eigenvalues with negative real part.

\section{B. Reduced Observer}

Clearly when measuring $y=C x$, it appears that we only need to reconstruct $z=H x$ where $H$ is chosen such that $\left(C^{T} H^{T}\right)^{T}$ has full column rank. Let us assume that $C$ has full row rank $p$. This amounts to stating that there are no redundant measurements in the output $y$. We can then find $H \in \mathbb{R}^{(n-p) \times n}$ such that

$$
\left(\begin{array}{l}
y \\
z
\end{array}\right)=\left(\begin{array}{l}
C \\
H
\end{array}\right) x \text { and } x=(S T)\left(\begin{array}{l}
y \\
z
\end{array}\right) .
$$

The partial state $z$ satisfies the differential equation

$$
\dot{z}(t)=H A T z(t)+H A S y(t) .
$$

It can be shown that under the assumption that the matrix pair $(A, C)$ be detectable that $H$ can be chosen such that 
$H A T$ is asymptotically stable. A reduced order observer is then given by

$$
\begin{aligned}
& \dot{\hat{z}}(t)=H A T \hat{z}(t)+H A S y(t) \\
& \hat{x}(t)=T \hat{z}(t)+S y(t) .
\end{aligned}
$$

Notice that if the matrix pair $(A, C)$ is not observable, but detectable, it may be that a reduced observer of lower dimension than $n-p$ exists, eg. in the case that $A$ is asymptotically stable, one could use as reduced observer $\hat{x} t \equiv 0$.

Let us finalize this section on observer design/synchronization for linear systems with the observation that the above ideas may be applied in the nonlinear context when we are interested in the neighborhood of an hyperbolic and stable fixed point to yield local results in observer/synchronization theory. However this is of limited value. Stability is essential as otherwise we are not guaranteed that the solutions will remain in a small neighborhood of the fixed point. Hyperbolicity is needed in order that the linearization captures the local behavior. This leads to a trivial observer problem, as the system is trivially detectable, setting the state estimate equal to the fixed point is an allowable observer strategy! This situation is unsatisfactory, and hence the need to have a more global point of view. This will be considered in the following sections. In the case where control can be applied to guarantee local stability, the linear perspective may be sufficient, however control aspects do complicate the discussion considerably.

\section{Linear Time Varying Systems}

Apart from nontrivial technical details, the situation for observer design in the case of linear time varying systems resembles strongly the theory for linear time invariant systems. We refer the interested reader to [20]. In [21], an application of a time varying linear system observer in the context of controlling chaos can be found. Time varying linear systems arise naturally when considering the linearization of nonlinear behavior in the neighborhood of a periodic orbit. They are also a standard tool in the context of extended Kalman filters.

\section{SySTEMS WITH LINEARIZABLE ERROR DyNAMICS}

From the previous examples a straightforward, yet nontrivial extension toward nonlinear systems transpires. The idea is to consider systems that may give rise to linear error dynamics perhaps via an appropriate change of coordinates and/or rescaling of the output variables. We first present a class of systems characterized by the fact that the nonlinearity only depends on the available output. Then we introduce the general idea. Next we present some fairly complete results to decide if a given system may give rise to linear error dynamics after suitable coordinate transformation. Again we treat the full observer case first, then the reduced observer.

\section{A. Output Only Nonlinearities}

When the only nonlinear terms in the system dynamics are function of the output only, the ideas pertaining to linear systems apply immediately.

Example 1: Consider the $n$ th-order nonlinear, time dependent differential equation in the scalar output variable $y$ :

$$
\begin{aligned}
\frac{d^{n}}{d t^{n}} y(t) & +\frac{d^{n-1}}{d t^{n-1}} f_{1}(y(t), t)+\cdots+\frac{d}{d t} f_{n-1}(y(t), t) \\
& +f_{n}(y(t), t)=0 .
\end{aligned}
$$

Assume that the functions $f_{i}$ have sufficient regularity to guarantee the existence of unique solutions on $\mathbb{R}$.

A particular state space realization for this differential equation (16) is obviously

$$
\begin{aligned}
\dot{x}(t) & =E x(t)+f(C x(t), t), \\
y(t) & =C x(t), \\
E & =\left(\begin{array}{lllll}
0 & 1 & 0 & & 0 \\
0 & 0 & 1 & & 0 \\
\vdots & & & \ddots & \\
0 & 0 & & & 1 \\
0 & 0 & & \cdots & 0
\end{array}\right) \in \mathbb{R}^{n \times n}, \\
C & =\left(\begin{array}{llll}
1 & 0 & \cdots & 0
\end{array}\right) \in \mathbb{R}^{1 \times n}, \\
f(y, t) & =\left(\begin{array}{llll}
-f_{1}(y, t) & -f_{2}(y, t) & \cdots & \left.-f_{n}(y, t)\right)^{T} .
\end{array}\right.
\end{aligned}
$$

Because the matrix pair $(E, C)$ is observable, and due to the specific structure of the state equation (17), it is obvious how to construct a full observer yielding asymptotically stable linear error dynamics

$$
\begin{aligned}
& \dot{\hat{x}}(t)=E \hat{x}(t)+f(y(t), t)+K(\hat{y}(t)-y(t)), \\
& \hat{y}(t)=C \hat{x}(t) .
\end{aligned}
$$

It suffices to choose $K$ such that $E+K C$ is asymptotically stable.

A generalization of the above example is the class of systems of Lur'e type, considered in, e.g., [38], [31], otherwise known as the output injection case

$$
\begin{aligned}
& \dot{x}(t)=A x(t)+f(C x(t), t), \quad x(0)=x_{0}, \quad t \geq 0 \\
& y(t)=C x(t) .
\end{aligned}
$$

Here $A, C$ are constant matrices of appropriate dimensions. Suppose that the solutions of (19) are well defined on $[0,+\infty)$. Assuming that the matrix pair $(A, C)$ is detectable, a full observer system takes the form:

$$
\begin{aligned}
\dot{\hat{x}}(t) & =A \hat{x}(t)+f(y(t), t)+K(\hat{y}(t)-y(t)) \\
\hat{x}(0) & =\hat{x}_{0}, \quad t \geq 0 \\
\hat{y}(t) & =C \hat{x}(t) .
\end{aligned}
$$

It suffices to choose $K$ such that $A+K C$ is asymptotically stable.

In [38], the authors consider the case where $A$ is asymptotically stable. This allows one to choose $K=0$ in (20). The advantage of $K$ is of course that we can consider the case where $A$ is not stable and that we can affect the error 
convergence. Well known examples of systems that fall into this category are the Chua circuit, the Duffing equation and van der Pol oscillator. We present a few examples.

Example 2: Consider the Duffing equation with periodic driving term

$$
\begin{aligned}
\dot{x}_{1}(t) & =x_{2}(t) \\
\dot{x}_{2}(t) & =-p_{1} x_{2}(t)-p_{2} x_{1}(t)-p_{3} x_{1}^{3}(t)+q \cos (\omega t) \\
y(t) & =x_{1}(t) .
\end{aligned}
$$

The solutions of (21) are well defined on $(0,+\infty)$ for any initial condition, provided $p_{1}, p_{2}, p_{3}>0$. This can easily be verified by considering the derivative of the comparison function $V=x_{2}^{2}+\left(p_{3} / 2\right) x_{1}^{4}+p_{2} x_{1}^{2}$ along the solutions of (21). It is well known that the solutions may exhibit chaos for particular parameter combinations.

This equation (21) is in the form of (19). With obvious definitions, the matrix pair $(A, C)$ is observable, and a full observer may be constructed as indicated in (20). The error $e(t)=x(t)-\hat{x}(t)$ satisfies

$$
\dot{e}(t)=(A+K C) e(t)=\left(\begin{array}{cc}
k_{1} & 1 \\
k_{2}-p_{2} & -p_{1}
\end{array}\right) e(t) .
$$

By an appropriate selection of the gain $K=\left(\begin{array}{ll}k_{1} & k_{2}\end{array}\right)^{T}$ the error dynamics (22) can be made asymptotically stable.

Example 3: Chua's circuit [11] can be described by the following state equations:

$$
\begin{aligned}
\frac{d}{d t}\left(\begin{array}{l}
x_{1}(t) \\
x_{2}(t) \\
x_{3}(t)
\end{array}\right)= & \left(\begin{array}{ccc}
-\frac{G}{C} & \frac{G}{C} & 0 \\
\frac{G}{Q} & -\frac{G}{Q} & \frac{1}{Q} \\
0 & -\frac{1}{L} & 0
\end{array}\right)\left(\begin{array}{l}
x_{1}(t) \\
x_{2}(t) \\
x_{3}(t)
\end{array}\right) \\
& +\left(\begin{array}{c}
-\frac{1}{C} f\left(x_{1}(t)\right) \\
0 \\
0
\end{array}\right) \\
y(t)= & \left(\begin{array}{lll}
1 & 0 & 0
\end{array}\right) x(t) .
\end{aligned}
$$

Clearly this is of the form of (19). The nonlinearity $f$ in (23) is given by $f(y)=Q_{1} y+1 / 2\left(Q_{2}-Q_{1}\right)\left(\left|y+y_{b}\right|-\left|y-y_{b}\right|\right)$ for some positive $Q_{1}, Q_{2}$ and $y_{b}$. This nonlinearity is not smooth, but this does not affect the discussion. Because $|f(y)| \leq \gamma|y|$ for some $\gamma>0$ it is easily shown that the solutions for all initial conditions are well defined on $\mathbb{R}$.

Clearly the linear part of the system equations (23) is observable. An observer may thus be constructed as before

$$
\begin{aligned}
\frac{d}{d t}\left(\begin{array}{c}
\hat{x}_{1}(t) \\
\hat{x}_{2}(t) \\
\hat{x}_{3}(t)
\end{array}\right)= & \left(\begin{array}{ccc}
-\frac{G}{C} & \frac{G}{C} & 0 \\
G & -\frac{G}{Q} & \frac{1}{Q} \\
\bar{Q} & -\frac{1}{Q} & 0
\end{array}\right)\left(\begin{array}{l}
\hat{x}_{1}(t) \\
\hat{x}_{2}(t) \\
\hat{x}_{3}(t)
\end{array}\right) \\
& +\left(\begin{array}{c}
-\frac{1}{C} f(y(t)) \\
0 \\
0
\end{array}\right)+\left(\begin{array}{l}
k_{1} \\
k_{2} \\
k_{3}
\end{array}\right)(\hat{y}(t)-y(t)) \\
\hat{y}(t)= & \left(\begin{array}{lll}
1 & 0 & 0
\end{array}\right) \hat{x}(t) .
\end{aligned}
$$

As in the previous Example 2 the $A$-matrix is asymptotically stable. The choice $k_{1}=k_{2}=k_{3}=0$ will yield asymptotically stable linear error dynamics [11]. Using different gains allows us to select a faster error response.

\section{B. Full Observer}

Starting from systems of the form (5) with output $y(t)=$ $h(x(t))$, a more general idea is to find a coordinate transformation $\xi=\phi(x)$ and output transformation $\eta=\psi(y)$ such that in the new coordinates we have a system description of the form

$$
\begin{aligned}
& \dot{\xi}(t)=A \xi(t)+g(\eta(t), t), \quad \xi(0)=\xi_{0}, \quad t \geq 0 \\
& \eta(t)=C \xi(t) .
\end{aligned}
$$

Provided the matrix pair $(A, C)$ is detectable we can construct an observer which gives rise to linear error dynamics in the usual way.

Notice that although we may obtain error dynamics which are seemingly defined on $\mathbb{R}$, this does not imply that the observer problem is well defined. The solutions to (25) have to be defined on $(0,+\infty)$ before it makes sense to discuss the error dynamics.

Some examples may serve to illustrate the basic idea.

Example 4: Let us consider the Rössler system:

$$
\begin{aligned}
\frac{d}{d t}\left(\begin{array}{l}
x_{1}(t) \\
x_{2}(t) \\
x_{3}(t)
\end{array}\right) & =\left(\begin{array}{c}
-x_{2}(t)-x_{3}(t) \\
x_{1}(t)+a x_{2}(t) \\
c+x_{3}(t)\left(x_{1}(t)-b\right)
\end{array}\right) \\
y & =\left(\begin{array}{lll}
0 & 0 & 1
\end{array}\right) x
\end{aligned}
$$

In the above (26) the coefficients $a, b, c>0$. Assume also that $x_{3}(0)>0$, then $x_{3}(t)=y(t)>0$ for all $t \geq 0$. Keeping this restriction in mind we may use the comparison function $V=1 / 2\left(x_{1}^{2}+x_{2}^{2}+x_{3}\right)>0$. Taking the derivative along the solutions of (26) $\dot{V}=a x_{2}^{2}+c-b x_{3} \leq a V+c$ which implies that the solutions (with $x_{3}(0)>0$ ) are well defined on $(0,+\infty)$. The observer problem is hence well posed.

We introduce now the following coordinates:

$$
\left(\begin{array}{lll}
\xi_{1} & \xi_{2} & \xi_{3}
\end{array}\right)=\left(\begin{array}{lll}
x_{1} & x_{2} & \ln \left(x_{3}\right)
\end{array}\right), \quad \eta=\ln y .
$$

In the new coordinates the system equations are then given by

$$
\begin{aligned}
\frac{d}{d t}\left(\begin{array}{l}
\xi_{1}(t) \\
\xi_{2}(t) \\
\xi_{3}(t)
\end{array}\right)= & \left(\begin{array}{rrr}
0 & -1 & 0 \\
1 & a & 0 \\
1 & 0 & 0
\end{array}\right)\left(\begin{array}{l}
\xi_{1}(t) \\
\xi_{2}(t) \\
\xi_{3}(t)
\end{array}\right) \\
& +\left(\begin{array}{c}
-e^{\xi_{3}(t)} \\
0 \\
-b+c e^{-\xi_{3}(t)}
\end{array}\right) \\
\eta(t)= & \left(\begin{array}{lll}
0 & 0 & 1
\end{array}\right) \xi(t) .
\end{aligned}
$$

The linear part of (28) is again observable and hence an observer with linear, asymptotically stable dynamics may be constructed as before. 
Example 5: The hyper chaotic Rössler system can be treated in a similar way, see also [36]

$$
\begin{aligned}
\frac{d}{d t}\left(\begin{array}{l}
x_{1}(t) \\
x_{2}(t) \\
x_{3}(t) \\
x_{4}(t)
\end{array}\right) & =\left(\begin{array}{c}
-x_{2}(t)-x_{3}(t) \\
x_{1}(t)+0.25 x_{2}(t)+x_{4}(t) \\
3+x_{3}(t) x_{1}(t) \\
-0.5 x_{3}(t)+0.05 x_{4}(t)
\end{array}\right) \\
y & =\left(\begin{array}{llll}
0 & 0 & 1 & 0
\end{array}\right) x=x_{3} .
\end{aligned}
$$

Assume also that $x_{3}(0)>0$ then $x_{3}(t)=y(t)>0$ for all $t \geq 0$. Let us restrict ourselves to the domain in which the chaotic attractor exists. With a coordinate transformation similar to what was introduced in the Example 4

$$
\begin{gathered}
\left(\begin{array}{cccc}
\xi_{1} & \xi_{2} & \xi_{3} & \xi_{4}
\end{array}\right)=\left(\begin{array}{ccc}
x_{1} & x_{2} \quad \ln \left(x_{3}\right) & x_{4}
\end{array}\right) \\
\eta=\ln y
\end{gathered}
$$

we may proceed as in the Example 4.

We now present for time invariant systems of the form (1) with scalar output $y$ conditions that allow one to transform via appropriate coordinate changes the system (1) into a system of the form (25). The more general case of multiple outputs has also been treated in [15], [27], and [28].

We consider the situation where the transformation from (1) to (25) only involves a state space coordinate change $\xi=\phi(x)$. This result is discussed in detail in [4]. Let $n$ be the state dimension of (1). The conditions under which the transformation may be achieved are given by the following.

1) Local observability

$$
\operatorname{dim}\left(\operatorname{span}\left\{D h(x), D L_{f} h(x), \cdots D L_{f}^{n-1} h(x)\right\}\right)=n, \forall x .
$$

2) The mapping defined as

$$
\xi:=\phi(x):=\left(h(x) L_{f} h(x) \cdots L_{f}^{n-1} h(x)\right)^{T}
$$

is a global diffeomorphism on $\mathbb{R}^{n}$.

3) The unique vector field $r$ on $\mathbb{R}^{n}$ that satisfies

$$
\begin{aligned}
L_{r} h(x) & =L_{r} L_{f} h(x)=\cdots=L_{r} L_{f}^{n-2} h(x)=0, \\
L_{r} L_{f}^{n-1} h(x) & =1,
\end{aligned}
$$

is such that

$$
\left[r, a d_{f}^{k} r\right]=0 \quad \forall k=1,3,5, \cdots, 2 n-1 .
$$

A necessary and sufficient result which involves both a state space transformation $\xi=\phi(x)$ as well as an output transformation $\eta=\psi(y)$ is discussed in [15]. See also [26]-[28].

\section{Reduced Observer}

The above result(s) are clearly relevant for the full observer problem. If a full observer with linear error dynamics may be found, a reduced observer with linear error dynamics can also be constructed. The reverse may not be the case. As far as we are aware, no results are available that provide conditions, under which via a state transformation and output transformation, a reduced observer with linear error dynamics may be found.
The Examples 2-4 readily allow for reduced observers to be found that yield linear error dynamics. After the coordinate transformation the methods valid for linear systems apply immediately.

Let us give an example for which the conditions (31)-(34) are not all satisfied, yet the system is such that we are still able to solve the reduced observer problem with error dynamics which are linear.

Example 6: Consider the van der Pol equation with driving term.

$$
\begin{aligned}
\dot{x}_{1}(t) & =x_{2}(t) \\
\dot{x}_{2}(t) & =\mu\left(1-x_{1}^{2}(t)\right) x_{2}(t)-x_{1}(t)+q \cos (\omega t) \\
y(t) & =x_{1}(t) .
\end{aligned}
$$

The parameter $\mu$ is positive. It is well known that the solutions of (35) are well defined on $\mathbb{R}^{+}$and that they may exhibit chaotic behavior for certain parameter selections $(\mu, q$ and $\omega)$.

It is easily verified that (31) is satisfied. Moreover the constant vector field $r(x)=\left(\begin{array}{ll}0 & 1\end{array}\right)^{T}$ satisfies (33) but fails to satisfy (34). Hence it is not possible to produce via a state coordinate transformation alone linear error dynamics for an observer.

However a reduced order observer with linear error dynamics can be found. Consider the new variable $z=x_{2}+k_{1} y+$ $k_{2} y^{3}$. It satisfies the differential equation

$$
\begin{aligned}
\dot{z}(t)= & \left(\mu+k_{1}\right) x_{2}(t)+\left(3 k_{2}-\mu\right) x_{1}^{2}(t) x_{2}(t)-x_{1}(t) \\
& +q \cos (\omega t) .
\end{aligned}
$$

Selecting $k_{1}+\mu=-1$ and $3 k_{2}-\mu=0$ we get

$$
\dot{z}(t)=-z(t)+(-2-\mu) y(t)+\frac{\mu}{3} y^{3}(t)+q \cos (\omega t) .
$$

This suggest the reduced observer

$$
\begin{aligned}
\dot{\hat{z}}(t) & =-\hat{z}(t)+(-2-\mu) y(t)+\frac{\mu}{3} y^{3}(t)+q \cos (\omega t) \\
\hat{x}_{2}(t) & =\hat{z}(t)+(1+\mu) y(t)-\frac{\mu}{3} y^{3}(t) \\
\hat{x}_{1}(t) & =y(t)
\end{aligned}
$$

The corresponding error dynamics are $\dot{e}_{r}(t)=-e_{r}(t)$, where $e_{r}=z-\hat{z}$.

Example 7: In [34], the following hysteretic circuit is described:

$$
\begin{aligned}
\dot{x}_{1}(t) & =x_{2}(t)+g x_{1}(t)+c x_{3}(t) \\
\dot{x}_{2}(t) & =-\omega x_{1}(t)+d_{2} x_{2}(t) \\
\epsilon \dot{x}_{3}(t) & =\left(1-x_{3}^{2}(t)\right)\left(S x_{1}(t)-D+x_{3}(t)\right)-d_{3} x_{3}(t) .
\end{aligned}
$$

The parameters $g, c, \omega, d_{2}, S, D, d_{3}$ and $\epsilon$ are all assumed to be positive. The output (described in [8] as the drive signal) is given by $y=x_{3}+\beta x_{1}+\alpha x_{2}$. The particular output chosen in [8] had $\alpha=0$. Expressing (39) in terms of the equivalent coordinates $(v, w, y)=\left(x_{1}, x_{2}, y\right)$ we obtain

$$
\begin{aligned}
\dot{v}(t) & =(1-c \alpha) w(t)+(g-c \beta) v(t)+c y(t) \\
\dot{w}(t) & =-\omega v(t)+d_{2} w(t) \\
\epsilon \dot{y}(t) & =F(v(t), w(t), y(t)) .
\end{aligned}
$$


The subsystem formed by $(v, w)$ is linear, time-invariant and can be rendered stable by selecting $\beta, \alpha$ appropriately (e.g., $g=c \beta$ and $\alpha=0$ ). It is therefore suitable to construct a reduced observer. In actual fact, we have full control over the speed of convergence of the reduced observer through tuning the parameters $\alpha, \beta$.

\section{Linear Time Varying Error Dynamics}

In the above examples we were lead to time invariant error dynamics. This may not always be achievable, but it may be possible to attain linear time varying error dynamics. For a more complete discussion we refer to [29].

Example 8: Let us reconsider the van der Pol equation with driving term (35), but this time we want to obtain a full observer.

Design the observer to be of the form

$$
\begin{aligned}
\dot{\hat{x}}_{1}(t)= & \hat{x}_{2}(t)+\alpha_{1}(y(t)) e_{1}(t) \\
\dot{\hat{x}}_{2}(t)= & \mu\left(1-y^{2}(t)\right) \hat{x}_{2}(t)-\hat{x}_{1}(t)+\left(\alpha_{2}(y(t))-1\right) e_{1}(t) \\
& +q \cos (\omega t) \\
\hat{y}(t)= & \hat{x}_{1}(t) \\
e(t)= & x(t)-\hat{x}(t)
\end{aligned}
$$

This gives rise to the following error dynamics

$$
\begin{aligned}
& \dot{e}_{1}(t)=e_{2}(t)-\alpha_{1}(y(t)) e_{1}(t) \\
& \dot{e}_{2}(t)=\mu\left(1-y^{2}(t)\right) e_{2}(t)-\alpha_{2}(y(t)) e_{1}(t) .
\end{aligned}
$$

The error dynamics, considering $y(t)$ as given, are linear time varying. In order to select the functions $\alpha_{1}$ and $\alpha_{2}$ we proceed with a Lyapunov analysis. As candidate Lyapunov function we propose $V\left(e_{1}, e_{2}\right)=(1 / 2)\left(e_{1}-(1 / 2 \mu) e_{2}\right)^{2}+(1 / 2) e_{1}^{2}$. For its derivative along the solutions of (42) we find

$$
\begin{aligned}
\dot{V}(t)= & -\frac{1}{4 \mu}\left(1+y^{2}(t)\right) e_{2}^{2}(t) \\
& +\left(-2 \alpha_{1}(y(t))+\frac{1}{2 \mu} \alpha_{2}(y(t))\right) e_{1}^{2}(t) \\
& +\left(\left(\frac{3}{2}+\frac{1}{2} y^{2}(t)\right)+\frac{1}{2 \mu} \alpha_{1}(y(t))-\frac{1}{4 \mu^{2}} \alpha_{2}(y(t))\right) \\
& \cdot e_{1}(t) e_{2}(t) .
\end{aligned}
$$

The following selection of $\alpha_{1}$ and $\alpha_{2}$ will make $\dot{V}<0$, which implies asymptotic stability for the error dynamics:

$$
\begin{aligned}
& \alpha_{1}(y)=\mu\left(3+y^{2}+\frac{1}{\mu^{2}}\right) \\
& \alpha_{2}(y)=2+4 \mu^{2}\left(3+y^{2}\right) .
\end{aligned}
$$

The method followed in solving the above example appears misleadingly straightforward, in general it is extremely hard to establish stability properties for linear time varying systems. Finding suitable Lyapunov functions is difficult, nevertheless Lyapunov theory is a very useful tool. For systems of the form $\dot{x}(t)=A(y(t)) x(t)+B(y(t))$ with $y=C x$, where $A$ depends in a smooth way on $y$ and such that the family of matrices $(A(y), C)$ is uniformly (in $y$ ) detectable, there is however a slightly more systematic design method for achieving a full order observer. It is also based upon Lyapunov theory. The observer may be constructed as

$$
\dot{\hat{x}}(t)=A(y(t)) \hat{x}(t)+B(y(t))+K(y(t)) y(t)
$$

where $K(y)=-R(y)^{-1} C^{T}$ and $R(y)$ is the unique symmetric positive definite solution of the Riccati equation [41]

$$
0=R(y) A(y)+A^{T}(y) R(y)+R(y) C^{T} C R(y)-C^{T} C .
$$

Examples where this technique may be applied are the Lorenz equations, as discussed in, e.g., [9], and Example 7 when considering a full observer and also the previous Example 8. This approach is non trivial as it requires one to solve (analytically) a Riccati equation which depends on a parameter $y$.

\section{General Systems}

As stated before providing conditions under which the general observer problem as stated in Section II may be solved, is very difficult. However, under the reasonable restriction that the dynamics are constrained to a compact domain, which is in particular the case when the system dynamics evolve on some strange attractor, a positive result using a high gain observer can be derived.

\section{A. High Gain Observer}

If we limit ourselves to dynamics defined on some compact set, then the following result is available [16].

Consider a time invariant system of the form (1). Let $p=1$. Assume that $\Omega \subset \mathbb{R}^{n}$ is a compact and a positively invariant set for the dynamics (1). Assume that $\xi:=\phi(x):=\left(h(x) L_{f} h(x) \cdots L_{f}^{n-1} h(x)\right)^{T}$ is a diffeomorphism on an open subset containing $\Omega$. The system equation (1) in the new coordinates $\xi$ are represented by

$$
\begin{aligned}
& \dot{\xi}(t)=F(\xi(t)) \\
& y(t)=\xi_{1}(t) .
\end{aligned}
$$

Consider also the system

$$
\begin{aligned}
& \dot{\hat{\xi}}(t)=F(\hat{\xi}(t))+K_{\theta}(\hat{y}(t)-y(t)) \\
& \hat{y}(t)=\hat{\xi}_{1}(t) .
\end{aligned}
$$

where the constant gain $K_{\theta} \in \mathbb{R}^{n}$ is defined via

$$
K_{\theta}=-S_{\theta}^{-1} C^{T}
$$

where $S_{\theta}=S_{\theta}^{T}>0$ solves

$$
0=\theta S_{\theta}+A^{T} S_{\theta}+S_{\theta} A-C^{T} C
$$

where

$$
C=\left(\begin{array}{llll}
1 & 0 & \cdots & 0
\end{array}\right) \in \mathbb{R}^{1 \times n}
$$

and

$$
A=\left(\begin{array}{cc}
0_{n-1 \times 1} & I_{n-1} \\
0 & 0_{1 \times n-1}
\end{array}\right) .
$$

The system (44) is an observer for the system (43) for all sufficiently large $\theta>0$, in that for all $\xi_{0}$ and all $\hat{\xi}_{0}$ in $\phi(\Omega)$, the error $e(t)=\xi(t)-\hat{\xi}(t)$ decreases exponentially. 
The equation $0=\theta S_{\theta}^{2}+A^{T} S_{\theta}+S_{\theta} A-C^{T} C$ to be solved for $S_{\theta}$ is known as an algebraic Riccati equation. The observability of $(A, C)$ guarantees the existence of a positive definite solution with the property that $A+K_{\theta} C$ is an asymptotically stable matrix.

The error dynamics are in general nonlinear, but due to the large gain $K_{\theta}$ the error dynamics on $\phi(\Omega)$ are essentially dominated by the stability of $A+K_{\theta} C$.

\section{B. Nonlinear Error Dynamics}

We conclude this section with one more example inspired by the Lorenz equations and which gives rise to a nonlinear observer with nonlinear error dynamics. We again exploit Lyapunov arguments [23], [24] to establish global asymptotic stability for the nonlinear error dynamics.

Example 9: Consider the dynamical system

$$
\begin{aligned}
\dot{x}_{1}(t) & =\sigma\left(x_{2}(t)-x_{1}^{3}(t)\right) \\
\dot{x}_{2}(t) & =\rho x_{1}(t)-x_{2}^{3}(t)-x_{1}(t) x_{2}(t) x_{3}(t) \\
\dot{x}_{3}(t) & =x_{1}(t) x_{2}^{2}(t)-\beta x_{3}(t) \\
y(t) & =x_{1}(t) .
\end{aligned}
$$

This system is not observable, but detectable for $\beta>0$ and $\sigma, \rho \neq 0$. Indeed computing the observability codistribution we obtain

$$
\begin{aligned}
d O(x)=\operatorname{span}\left\{(1,0,0),(*, \sigma, 0),\left(*, *, \sigma x_{1} x_{2}\right)\right. \\
\left.\left(*, *,-\sigma^{2} x_{2}^{2}-\rho \sigma x_{1}^{2}+\sigma x_{1} x_{2} g(x)\right), \cdots\right\} .
\end{aligned}
$$

Clearly if either $x_{1} \neq 0$ or $x_{2} \neq 0$ we have that $\operatorname{dim} d O(x)=3$. It can be seen that if $x_{1}=0$ and $x_{2}=0$ then $\operatorname{dim} d O(x)=2$ and the $\operatorname{ker} d O(x)=\left\{\left(0,0, x_{3}\right)\right\}$. Moreover this ker is invariant. Indeed $x_{1}(0)=0$ and $x_{2}(0)=0$ imply that $x_{1}(t, 0)=0, x_{2}(t, 0)=0$ for all $t$ and also that $\dot{x}_{3}(t)=-\beta x_{3}(t)$. The system is thus detectable, but not observable.

Consider the situation where the parameters $\rho, \sigma, \beta>0$. In this case the origin is a saddle point for the system (45). Using the comparison function $V=x_{1}^{2}+x_{2}^{2}+x_{3}^{2}$ it can be shown that the solutions are well defined on $\mathbb{R}^{+}$, and that the solutions are ultimately bounded. Indeed $\dot{V}=-\sigma x_{1}^{4}-x_{2}^{4}+(\rho+\sigma) x_{1} x_{2}-$ $\beta x_{3}^{2}$. It follows that $\dot{V} \leq 0$ outside some compact domain $\Omega$, let $M^{2}$ denote the maximum value that $V$ attains on $\Omega . \Omega$ is positively invariant.

We look for a reduced observer. Introduce the variable $z=\left(\begin{array}{ll}z_{1} & z_{2}\end{array}\right)^{T}$

$$
z_{1}(t)=x_{2}(t)-\frac{k}{\sigma} y(t) \quad z_{2}(t)=x_{3}(t)
$$

The partial state $z$ is governed by the differential equation

$$
\begin{aligned}
\dot{z}_{1}(t)= & -k\left(z_{1}(t)+k y(t)\right)-\left(z_{1}+k y(t)\right)^{3} \\
& -y(t)\left(z_{1}+k y(t)\right) z_{2}(t)-k y^{3}(t)+\rho y(t) \\
\dot{z}_{2}(t)= & y(t)\left(z_{1}(t)+k y(t)\right)^{2}-\beta z_{2}(t) .
\end{aligned}
$$

Choosing $k>M^{2}+\left(M^{4} / 2 \beta\right)$ leads to an exponentially stable observer given by

$$
\begin{aligned}
\dot{\hat{z}}_{1}(t)= & -k\left(\hat{z}_{1}(t)+k y(t)\right)-\left(\hat{z}_{1}(t)+k y(t)\right)^{3} \\
& -y(t)\left(\hat{z}_{1}(t)+k y(t)\right) \hat{z}_{2}(t)-k y^{3}(t)+\rho y(t) \\
\dot{\hat{z}}_{2}(t)= & y(t)\left(\hat{z}_{1}(t)+k y(t)\right)^{2}-\beta \hat{z}_{2}(t) .
\end{aligned}
$$

The error dynamics with $e_{1}=z_{1}-\hat{z}_{1}$ and $e_{2}=z_{2}-\hat{z}_{2}$ are governed by the nonlinear and time varying differential equation

$$
\begin{aligned}
\dot{e}_{1}(t)= & -k e_{1}(t)-e_{1}(t)\left[3 x_{2}^{2}(t)-3 x_{2}(t) e_{1}(t)+e_{1}^{2}(t)\right] \\
& -y(t) x_{2}(t) e_{2}(t)-y(t) x_{3}(t) e_{1}(t)+y(t) e_{1}(t) e_{2}(t) \\
\dot{e}_{2}(t)= & -\beta e_{2}(t)-y(t) e_{1}^{2}(t)+2 y(t) x_{2}(t) e_{1}(t) .
\end{aligned}
$$

Using the comparison function $W=e_{1}^{2}+e_{2}^{2}$ we can now establish that the error dynamics (48) have a uniformly asymptotically stable trivial solution provided $k>M^{2}+\left(M^{4} / 2 \beta\right)$.

\section{CONCLUSION}

We have drawn attention to the fact that the problem of synchronization as introduced in the control of chaos literature can be viewed as a special case of the observer design problem, which is well known in the nonlinear control theory literature. The formalism offered via the observer theory allows us to provide a reasonable comprehensive framework for synchronization issues. Some open research problems have been identified. We did not discuss the connections with the literature discussing the filtering problem.

This paper was preoccupied with the existence question. In a companion paper we will focus attention on the control problem formulation of synchronization [30] and in particular consider issues relating to sensitivity with respect to measurement errors and/or errors in the representation of the system dynamics. These are of great importance when considering observer/synchronization problems in a practical setting.

\section{REFERENCES}

[1] G. Chen, Control and Synchronization of Chaos, a Bibliography, Dept. Elect. Eng., Univ. Houston, Houston, TX, available via ftp:uhoop.egr.uh.edu/pub/TeX/chaos.tex (login name and password both "anonymous"), 1997.

[2] E. Ott, T. Sauer, and J. A. Yorke, Eds., Coping with Chaos: Analysis of Chaotic Data and the Exploitation of Chaotic Systems. New York: Wiley-Intersci., 1994.

[3] M. J. Ogorzałek, "Taming chaos-Part I: Synchronization," IEEE Trans. Circuits Syst. I, vol. 40, pp. 693-699, 1993.

[4] A. Isidori, Nonlinear Control Systems, 3rd ed. New York: SpringerVerlag 1995.

[5] H. Nijmeijer and A. van der Schaft, Nonlinear Dynamical Control Systems. New York: Springer-Verlag, 1990.

[6] M. Vidyasagar, Nonlinear Systems Analysis, 2nd ed. Englewood Cliffs, NJ: Prentice-Hall, 1993.

[7] T. L. Carroll and L. M. Pecora, "Synchronizing chaotic circuits," IEEE Trans. Circuits Syst., vol. 38, pp. 453-456, 1991.

[8] L. M. Pecora and T. L. Carroll, "Synchronization in chaotic systems," Phys. Rev. Lett., vol. 64, no. 8, pp. 821-824, 1990.

[9] K. Cuomo, A. Oppenheim, and S. Strogatz, "Robustness and signal recovery in a synchronized chaotic system," Int. J. Bifurc. Chaos, vol. 3, no. 6, 1993, pp. 1629-1638.

[10] R. He and P. Vaidya, "Analysis and synthesis of synchronous periodic and chaotic systems," Phys. Rev. A, vol. 46, no. 12, pp. 7387-7392, 1992. 
[11] C. W. Wu and L. Chua, "A simple way to synchronize chaotic systems with applications to secure communication systems," Int. J. Bifurc. Chaos, vol. 3, no. 6, pp. 1619-1627, 1993.

[12] ical systems," Int. J. Bifurc. Chaos, vol. 4, no. 4, 1994, pp. 979-998.

[13] D. G. Luenberger, Introduction to Dynamic Systems, Theory, Models and Applications. New York: Wiley, 1979.

[14] T. Kailath, Linear Systems. Englewood Cliffs, NJ: Prentice-Hall, 1980.

[15] K. Tchon and H. Nijmeijer, "On output linearization of observable dynamics," Contr. Theory Adv. Technol., vol. 9, no. 4, pp. 819-857, 1993.

[16] J. Gauthier, H. Hammouri, and S. Othman, "A simple observer for nonlinear systems, applications to bioreactors," IEEE Trans. Automat. Contr., vol. 37, pp. 875-880, 1992.

[17] F. Takens, "Detecting strange attractors in turbulence," Lecture Notes in Mathematics, vol. 898, pp. 366-381, 1981.

[18] D. Aeyels, "Generic observability of differentiable systems," SIAM J. Contr. Opt., vol. 19, pp. 595-603, 1981.

[19] __ "On the number of samples necessary to achieve observability," Syst. Contr. Lett., vol. 1, pp. 92-94, 1981.

[20] B. D. O. Anderson and J. B. Moore, "Detectability and stabilizability of time varying discrete time linear systems," SIAM J. Contr. Opt., vol. 19, no. 1, pp. 20-32, Jan. 1981.

[21] F. Romeiras, C. Grebogi, E. Ott, and W. Dayawansa, "Controlling chaotic dynamical systems," Physica D, vol. 58, no. 1-4, pp. 165-192, Sept. 15, 1992.

[22] J. K. Hale, Ordinary Differential Equations. Malabar, FL: Krieger, 1980.

[23] J. L. Willems, Stability Theory of Dynamical Systems. London, England: Nelson, 1970.

[24] W. Hahn, Stability of Motion. New York: Springer-Verlag, 1967.

[25] F. Romeiras, C. Grebogi, E. Ott, and W. Dayawansa, "Controlling chaotic dynamical systems," Physica D, vol. 58, no. 1-4 pp. 165-192, 1992.

[26] A. J. Krener and A. Isidori, "Linearization by output injection and nonlinear observers," Syst. Contr. Lett., vol. 3, pp. 47-52, 1983.

[27] A. Krener and J. Respondek, "Nonlinear observers with linearizable error dynamics," SIAM J. Contr. Opt., vol. 23, pp. 47-52, 1985.

[28] X. H. Xia and W. B. Gao, "Nonlinear observer design by observer error linearization," SIAM J. Contr. Opt., vol. 27, pp. 199-216, 1989.

[29] H. Hammouri and J. P. Gauthier, "Global timevarying linearization up to output injection," SIAM J. Contr. Opt., vol. 30, no. 6, pp. 1295-1310, Nov. 1992.

[30] I. I. Blekhman, A. L. Fradkov, H. Nijmeijer, and A. Y. Pogromsky, "On self-synchronization and controlled synchronization," Syst. Contr. Lett., 1997 , to be published.

[31] R. Genesio, A. Tesi, and F. Villoresi, "A frequency approach for analysing and controlling chaos in nonlinear circuits," IEEE Trans. Circuits Syst. II, vol. 40, pp. 819-828, 1993.

[32] T. L. Carroll and L. M. Pecora, "Synchronizing nonautonomous chaotic circuits," IEEE Trans. Circuits Syst. II, vol. 40, pp. 646-650, 1993.

[33] T .L. Carroll, J. F. Heagy, and L. M. Pecora, "Transforming signals with chaotic synchronization," Phys. Rev. E, vol. 54, no. 5, pp. 46776-4680, 1996.

[34] L. M. Pecora and T. L. Carroll, "Driving systems with chaotic signals," Phys. Rev. A, vol. 44, no. 4, pp. 23740-2383, 1991.

[35] M. Ding and E. Ott, "Enhancing synchronization of chaotic systems," Phys. Rev. E, vol. 49, no. 2, pp. 945-948, 1994.
[36] J. H. Peng, E. J. Ding, M. Ding, and W. Yang, "Synchronizing hyperchaos with a scalar transmitted signal," Phys. Rev. Lett., vol. 76, no. 6, pp. 904-907, 1996.

[37] T. Kapitaniak, L. O. Chua, and G. Q. Zhong, "Experimental synchronization of chaos using continuous control," Int. J. Bifurc. Chaos, vol 2, no. 6, pp. 483-488, 1994.

[38] A. Tesi, A. de Angeli, and R. Genesio, "On system decomposition for synchronizing chaos," Int. J. Bifurc. Chaos, vol. 4, no. 6, pp. 1675-1685, 1994.

[39] O. Morgül and E. Solak, "Observer based synchronization of chaotic signals," Phys. Rev. E, vol. 54, no. 5, pp. 4803-4811, 1996.

[40] K. M. Cuomo, A. V. Oppenheim, and S. H. Strogratz, "Synchronization of Lorenz-based chaotic circuits with application to communication," IEEE Trans. Circuits Syst. II, vol. 40, pp. 626-633, Oct. 1993.

[41] G. Besancon, "Contributions a l'etude et a l'observation des systemes non lineaire avec recour au calcul formel," Ph.D. dissertation, Institut National Polytechnique de Grenoble, 1996.

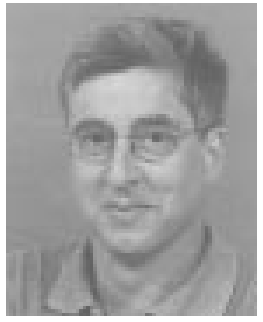

Henk Nijmeijer received the Ph.D. degree in 1983 from the University of Groningen, Groningen, The Netherlands

Since that year he has been with the Systems and Control Group of the Department of Applied Mathematics, University of Twente, The Netherlands, where he presently is an Associate Professor. His research interests are in nonlinear control and systems and applications.

Dr Nijmeijer is on the editorial board of various scientific journals including the SIAM Journal on Control and Optimization, the International Journal of Robust and Nonlinear Control, and Automatica.

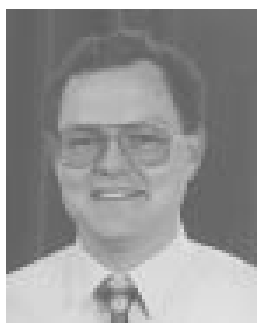

Iven M. Y. Mareels was born in Aalst, Belgium, in August 1959. He received the Bachelor of Engineering degree from the State Univeristy Gent, Gent, Belgium, in 1982 and the Ph.D. degree in systems engineering from the Australian National University, Canberra, Australia, in 1987.

From 1982 to 1987, and in 1988, he worked as a Research Associate with the Belgium National Fund for Scientific Research. He fulfilled his military service in 1987. From mid-1988 until the end of 1990, he was a Lecturer at Newcastle University, Newcastle, Australia. From January 1991 to July 1996, he was a Reader with the Department of Engineering, Australian National University. Since July 1996, he has been a Professor of electrical and electronic engineering, with the Department of Electrical and Electronic Engineering, The University of Melbourne, Melbourne, Australia. He currently heads that department. His research interest are in the general area of dynamical systems theory, modeling, and control of nonlinear systems.

Dr. Mareels is (together with Prof. A. Antoulas), Editor-in-Chief for the Journal Systems and Control Letters. 